\title{
Pax8 protein stability is controlled by sumoylation
}

\author{
Tiziana de Cristofaro, Anna Mascia, Andrea Pappalardo, Barbara D’Andrea, Lucio Nitsch \\ and Mariastella Zannini
}

Istituto di Endocrinologia e Oncologia Sperimentale 'G. Salvatore'-CNR and Dipartimento di Biologia e Patologia Cellulare e Molecolare, Università di Napoli Federico II, Via

S. Pansini 5, 80131 Napoli, Italy

(Correspondence should be addressed to M Zannini; Email: s.zannini@ieos.cnr.it)

\begin{abstract}
The transcription factor Pax8 is involved in the morphogenesis of the thyroid gland and in the maintenance of the differentiated thyroid phenotype. Despite the critical role played by Pax8 during thyroid development and differentiation, very little is known of its post-translational modifications and how these modifications may regulate its activity. We focused our attention on the study of a specific post-translational modification, i.e., sumoylation. Sumoylation is a dynamic and reversible process regulating gene expression by altering transcription factor stability, protein-protein interaction and subcellular localization of target proteins. The analysis of Pax8 protein sequence revealed the presence of one sumoylation consensus motif ( $\psi \mathrm{KxE})$, strongly conserved among mammals, amphibians, and fish. We demonstrated that Pax8 is sumoylated by the addition of a single small ubiquitin-like modifier (SUMO) molecule on its lysine residue 309 and that $\mathrm{Pax} 8^{\mathrm{K} 309 \mathrm{R}}$, a substitution mutant in which the candidate lysine is replaced with an arginine, is no longer modified by SUMO. In addition, we analyzed whether protein inhibitor of activated signal transducers and activators of transcription (PIASy), a member of the PIAS STAT family of proteins, could function as a SUMO ligase and we demonstrated that indeed PIASy is able to increase the fraction of sumoylated Pax8. Interestingly, we show that Pax8 is targeted in the SUMO nuclear bodies, which are structures that regulate the nucleoplasmic concentration of transcription factors by SUMO trapping. Finally, we report here that the steady-state protein level of Pax 8 is controlled by sumoylation.
\end{abstract}

Journal of Molecular Endocrinology (2009) 42, 35-46

\section{Introduction}

The transcription factor Pax8 is a member of the Pax family of genes encoding for DNA binding proteins that are involved in the regulation of the development of a variety of tissues in different species (Stuart \& Gruss 1996). Specifically, Pax 8 has been demonstrated to be required both for the morphogenesis of the thyroid gland and for the maintenance of the thyroid differentiated phenotype (Plachov et al. 1990, Poleev et al. 1992, Pasca di Magliano et al. 2000). Interestingly, in Pax8 knockout mice the thyroid gland is barely visible and lacks the follicular cells, the most abundant cell population of the thyroid gland (Mansouri et al. 1998). In addition, in these mice the expression of thyroid-specific markers, such as thyroglobulin $(\mathrm{Tg})$ and thyroperoxidase, cannot be detected. These in vivo studies led to the conclusion that Pax8 is needed for the proper differentiation of thyrocytes. Mutations in the Pax8 gene have also been associated with congenital hypothyroidism in humans (Macchia et al. 1998). Patients carrying the mutations are affected by thyroid dysgenesis, indicating an important role for this gene in thyroid organogenesis.

Despite the critical role played by Pax8 during thyroid development and differentiation, very little is known of its post-translational modifications and how these modifications may regulate its activity. Posttranslational modifications like phosphorylation, acetylation, ubiquitination, and sumoylation represent a major mechanism by which transcription factors activity is regulated in eukaryotes (Hunter 2007). Recent studies have demonstrated that the TSH regulates Pax8 transcriptional activity (Mascia et al. 2002), but there isn't exhaustive evidence of post-translational modifications (Poleev et al. 1992, Kambe \& Seo 1996).

In order to shed light on the mechanisms responsible for Pax8 post-translational regulation, we have investigated the role of sumoylation. Sumoylation is a dynamic and reversible process regulating (inhibiting or activating) gene expression by altering transcription factor stability, protein-protein interaction and subcellular localization of target proteins (Hay 2005, Heun 2007). Enzymes involved in small ubiquitin-like modifier (SUMO) conjugation have been identified both in yeast and in mammalian cells. While yeast and nematodes have a single SUMO gene, higher eukaryotes have three SUMO paralogues, SUMO-1, SUMO-2, SUMO-3, which are encoded by separate genes (Holmstrom et al. 2003). The mature forms of SUMO2 and SUMO-3 are very similar, but less closely related to SUMO-1. Despite their close similarity, there is evidence 
that SUMO-1 and SUMO-2/3 are preferentially conjugated to distinct sets of target proteins. Furthermore, mouse embryos lacking SUMO-1 die, indicating that SUMO-2/3 cannot compensate for SUMO-1 deficiency. The small ubiquitin-related modifier SUMO-1 covalently modifies a growing list of proteins including c-Jun (Muller et al. 2000), cAMP response element-binding protein (Creb1; Kuo et al. 2005), Sp3 (Ross et al. 2002), GATA-1 $\times$ (Collavin et al. 2004), glucocorticoid receptor (Tirard et al. 2007), c-Myc (Dahle et al. 2003), p300/CBP (Girdwood et al. 2003), and BMAL1 (Cardone et al. 2005).

The SUMO conjugation system is analogous to ubiquitination and involves machinery consisting of an E1-activating enzyme, an E2-conjugating enzyme (Ubc9), three different groups of E3-ligases to conjugate and several SUMO proteases to deconjugate SUMO from target proteins (Hay 2007, Zhao 2007). Whereas ubiquitination primarily mediates protein degradation, sumoylation appears to be involved in the regulation of diverse protein function such as gene expression, chromatin structure, nucleocytoplasmic traffic, and signal transduction (Melchior 2000, Muller et al. 2001, Seeler \& Dejean 2003).

In the present study, we report that $\operatorname{Pax} 8$ is conjugated to SUMO and we map the single lysine residue involved in the modification. We also demonstrate that protein inhibitors of activated signal transducers and activators of transcription (PIAS STAT; Schmidt \& Muller 2003, Shuai \& Liu 2005), functions as a SUMO ligase for Pax8 and enhances Pax8 sumoylation. In addition, we show that Pax8 is targeted to the SUMO nuclear bodies (SNBs; Navascues et al. 2007) and that sumoylation affects the protein stability of Pax8.

\section{Materials and methods}

\section{Plasmid constructs}

The plasmids used have been previously described and were as follows: CP5-CAT (Missero et al. 1998) CMV5-Pax8 (Zannini et al. 1992) and $3 \times$ FLAG-Pax8 (Di Palma et al. 2003). The mutants $\mathrm{Pax} 8^{\mathrm{K} 309 \mathrm{R}}, \operatorname{Pax} 8^{\mathrm{K} 2}, \operatorname{Pax} 8^{\mathrm{K} 91 \mathrm{R}}$ were constructed using the Quick Change XL Site-Directed Mutagenesis kit (Stratagene, La Jolla, CA, USA) as template to the CMV5-Pax8 construct. $3 \times$ FLAG-SUMO1 and $3 \times$ FLAG-SUMO- $\Delta$ gly constructs were generated by PCR amplification of SUMO-1 rat cDNA using specific primers: SUMO-1Fw: 5'CCCAAGCTTGGGATGTCTGACCAGGAGGCAAAACCTTC3'， SUMO-1Rev: 5'CGGGGTACCCCGCTAAACCGTCGAGTGACCCCCCGT3', and SUMO-1rev-mut: $5^{\prime}$ cggggtacccegctaaaccgtcgagtgagccgccgt $3^{\prime}$ and subsequent subcloning in the HindIIIKpnI sites of p3XFLAG-CMV ${ }^{\mathrm{TM}-10}$ expression vector
(Sigma, St Louis, MO, USA). The pCMV-T7-PIASy construct was kindly provided by Prof. Giannino del Sal.

\section{Cell culture and transfection}

PC Cl3 cells (Berlingieri et al. 1988) were grown in Coon's modified F-12 medium (Euroclone, Siziano, PV, Italy) supplemented with $5 \%$ calf serum and a six-hormone mixture $(6 \mathrm{H})$ as described by Ambesi-Impiombato \& Coon (1979).

HeLa cells were grown in DMEM (Euroclone) supplemented with 10\% FCS (Hyclone Logan, Utah, USA). For transient transfection experiments, cells were plated at $3 \times 10^{5}$ cells $/ 60$-mm tissue culture dish 10 to $12 \mathrm{~h}$ prior to transfection. Transfections were carried out with the FuGENE6 reagent (Roche Diagnostics, Mannheim, Germany) according to the manufacturer's directions. The DNA/FuGENE ratio was $1: 3$ in all the experiments.

\section{Transactivation assays}

The plasmid CMV-LUC was used as the internal control and the total amount of transfected DNA was kept constant with an empty expression vector in all the transfection assays. Cell extracts were prepared $48 \mathrm{~h}$ after transfection to determine either the levels of the CAT protein with a CAT ELISA kit (Roche Diagnostics) or the LUC activity by a luciferase assay as previously described (de Wet et al. 1987). Transfection experiments were done in duplicate and repeated at least three times. CAT activity values on the graph are the means of all experiments $( \pm)$ s.D. Statistical analysis has been performed by means of an unpaired two-tailed Student's $t$-test to obtain the $P$ value associated with the observed fold of activation differences.

\section{Protein extracts and immunoblotting}

For reporter assays, cells were washed twice with ice-cold PBS and lysed in a buffer containing $10 \mathrm{mM}$ Hepes $\mathrm{pH}$ $7 \cdot 9,400 \mathrm{mM} \mathrm{NaCl}, 0 \cdot 1 \mathrm{mM}$ EGTA pH 7·8, 5\% glycerol, $1 \mathrm{mM}$ DTT, $1 \mathrm{mM}$ phenylmetilsulfonil fluoride (PMSF).

The protein concentration was determined using the Bio-Rad protein assay (Bio-Rad Laboratories Inc, Hercules, CA, USA).

For sumoylation assay, cells were washed in PBS and disrupted in SDS-Laemmli buffer with $10 \mathrm{mM}$ $N$-ethylmaleimide (NEM) and boiled at $95^{\circ} \mathrm{C}$. Then the samples were analyzed by western blot.

For anti-T7-tag antibody co-immunoprecipitation, cells were harvested $48 \mathrm{~h}$ after transfection in $200 \mu \mathrm{l}$ buffer, $150 \mathrm{mM} \mathrm{NaCl}, 50 \mathrm{mM}$ Hepes at $\mathrm{pH} \mathrm{7 \cdot 5,10 \%}$ glycerol, 0,1\% Tween 20, $10 \mathrm{mM}$ NEM, $1 \mathrm{mM}$ PMSF, and a complete protease inhibitor cocktail tablet 
(Roche). Cell debris was removed by centrifugation for $30 \mathrm{~min}$. Lysates were first cleared with protein $\mathrm{G}$ beads for $30 \mathrm{~min}$, followed by incubation with antibody for $1 \mathrm{~h}$ at $4{ }^{\circ} \mathrm{C}$. Finally, the antibody complexes were captured with protein $\mathrm{G}$ beads for $1 \mathrm{~h}$. Beads were washed four times with the same buffer and immunoprecipitates were analyzed by western blot.

For purification on anti-FLAG M2 agarose affinity gel (Sigma), $48 \mathrm{~h}$ after transfection cells were washed twice with PBS and lysed in RIPA buffer $(150 \mathrm{mM} \mathrm{NaCl}$, $10 \mathrm{mM}$ Tris-HCl pH 7·2, 0,1\% SDS, $1 \%$ TRITON X-100, $1 \%$ sodium deoxycholate, $5 \mathrm{mM}$ EDTA pH $8,10 \mathrm{mM}$ NEM, $1 \mathrm{mM}$ PMSF, and a complete protease inhibitor cocktail tablet). $1.5 \mathrm{mg}$ total protein extract was purified on agarose affinity gel for $6 \mathrm{~h}$ at $4{ }^{\circ} \mathrm{C}$ on a rotating wheel. Bound proteins were eluted by resuspending the beads directly in 2X SDS-PAGE sample buffer and heating at $95^{\circ} \mathrm{C}$ for $5 \mathrm{~min}$.

For western blot analysis, proteins were separated on SDS- $10 \%$ or $12 \%$ PAGE, gels were blotted onto Immobilon P (Millipore, Bredford, MA, USA) for $2 \mathrm{~h}$ and the membranes were blocked in 5\% non-fat dry milk in Tris-buffered saline for $2 \mathrm{~h}$ or overnight before the addition of the antibody for $1 \mathrm{~h}$. The primary antibodies used were anti-PAX8 (kindly provided by Di Lauro), anti-tubulin (Santa Cruz Biotechnology Inc, Santa Cruz, CA, USA), anti-FLAG (Sigma) and anti-T7 (Novagen, Madison, WI, USA). The filters were washed three times in Tris-buffered saline plus $0.5 \%$ Tween 20 before the addition of HRP-conjugated secondary antibodies for $45 \mathrm{~min}$. HRP was detected with ECL (GE Healthcare, Waukesha, WI, USA).

\section{Immunofluorescence analysis}

HeLa and PC Cl3 cells were plated and cultured on $12 \mathrm{~mm}$ diameter glass coverslips 24 to $48 \mathrm{~h}$ prior to transfection with FuGENE6 reagent. Transfections were performed with $500 \mathrm{ng}$ of each plasmid used and DNA/FuGENE ratio was 1:6 in all the experiments. Twenty-four hours after transfection, cells were fixed for $20 \mathrm{~min}$ at room temperature with a $4 \%$ solution of paraformaldehyde in PBS, treated for $20 \mathrm{~min}$ with $50 \mathrm{mM}$ solution of $\mathrm{NH}_{4} \mathrm{Cl}$ in PBS, permeabilized for 5 min with a $0.5 \%$ solution of Triton X-100 in PBS and incubated for $60 \mathrm{~min}$ in $1 \%$ BSA in PBS. The coverslips were subsequently incubated for $60 \mathrm{~min}$ at room temperature with primary antibodies diluted in $1 \%$ BSA in PBS. After PBS washings, the coverslips were incubated for 30 min with Alexa 594-tagged goat antirabbit, and Alexa 488-tagged goat anti-mouse secondary antibodies diluted 1:200 in 1\% BSA in PBS. After final washings with PBS, the coverslips were mounted on a microscope slide using a $50 \%$ solution of glycerol in PBS.

Primary antibody dilutions were the following: mouse monoclonal anti-flag 1:1000 (Sigma); rabbit polyclonal
anti-Pax8 1:1000; mouse monoclonal anti-promyelocitic leukemia protein (PML; PG-M3) 1:200 (Santa Cruz). Confocal scanning laser microscopy images were collected with a Zeiss LSM 510 confocal laser scanning microscope, equipped with a $543 \mathrm{~nm} \mathrm{HeNe}$ laser, a $488 \mathrm{~nm}$ argon ion laser and a Plan-Apochromat $63 \times$ $/ 1 \cdot 4$ oil-immersion objective. Emitted fluorescence was detected using a BP 505-530 bandpass filter for Alexa Fluor 488 and a LP 560 long pass filter for Alexa Fluor 594. Pairs of images were collected simultaneously in the green and red channels. High magnification images were collected as $1024 \times 1024 \times 32$ voxel images.

\section{Electrophoretic mobility-shift assay}

Double-stranded oligonucleotides corresponding to the rat $\mathrm{Tg}$ promoter, 5'-CACTGCCCAGTCAAGTGTTCTTGA-3 $3^{\prime}$ were labeled with $\gamma-{ }^{32} \mathrm{P}$ ATP and T4 polynucleotide kinase and used as probes. The binding reactions were carried out in a buffer containing $10 \mathrm{mM}$ Hepes (pH 7.9), $10 \%$ glycerol, $0 \cdot 1 \mathrm{mM}$ EDTA, $8 \mathrm{mM}$ $\mathrm{MgCl}_{2}, 1 \mathrm{mM}$ DTT, $0 \cdot 15 \mu \mathrm{g} / \mathrm{ml}$ poly (dI-dC) for $30 \mathrm{~min}$ at room temperature. DNA-protein complexes were resolved on a $6 \%$ non-denaturing polyacrylamide gel and visualized by autoradiography.

\section{Results}

\section{Thyroid transcription factor Pax 8 is sumoylated}

Protein sumoylation occurs on the lysine of a $\psi \mathrm{KxE}$ protein motif (Song et al. 2004), where $\psi$ represents the hydrophobic residue isoleucine, leucine or valine and $\mathrm{x}$ is any amino acid. Analysis of the murine protein sequence of Pax8 (Genbank CAA40725) using the SUMO plot software from Abgent revealed the presence of two putative sumoylation sites at positions K91 and K309, representing a low score site $(0 \cdot 33)$ and an high score site $(0.94)$ respectively. In agreement with these results, the putative site containing the lysine residue 91 is conserved only in mammals (data not shown), while the high score site centered on lysine 309 is strongly conserved among mammals (human, Genbank NP_003457), amphibians (Xenopus laevis, Genbank AAD52681), and fish (zebrafish Genbank AAC31813; Fig. 1).

To test whether Pax8 could be a substrate for SUMO modification, we transiently co-transfected HeLa cells with the expression vectors encoding Pax 8 and $3 \times$ FLAG-SUMO-1. Protein extracts prepared from transfected cells were analyzed by western blot. As shown in Fig. 2A, an anti-Pax8 polyclonal antibody detected an additional slower-migrating band in the extract prepared from HeLa cells expressing Pax8 and FLAG-SUMO-1, which is compatible with a single 


$\begin{array}{lll}\text { Human } & \text { HSPFAIKQETPEVSSSS } & 319 \\ \text { Mouse } & \text { HSPFAIKQETPELSSSS } & 320 \\ \text { X. laevis } & \text { FTAFSIKQEASDSSSAS } & 334 \\ \text { Zebrafish } & \text { PLPLCLKQEVSPEVNSL } & 290 \\ & & \\ \text { Consensus motif } \psi \mathrm{KXE} & \end{array}$

Figure 1 Sequence alignment of the Pax8 region surrounding the site of SUMO attachment. Amino acids conforming to the consensus motif for sumoylation are highlighted, where $\psi$ represents the hydrophobic residue isoleucine, leucine or valine, and $\mathrm{x}$ is any amino acid. The consensus motif is strongly conserved among mammals, amphibians, and fish.

SUMO molecule attachment. This slower-migrating band was not visible when Pax8 was transfected alone. To investigate whether Pax8 lysine 309 might indeed serve as an acceptor site for SUMO conjugation, we generated the substitution mutant $\mathrm{Pax} 8^{\mathrm{K} 309 \mathrm{R}}$ in which the lysine was replaced with an arginine by site-directed mutagenesis. Transient transfection in HeLa cells with the expression vectors encoding for $\mathrm{Pax} 8^{\mathrm{K} 309 \mathrm{R}}$ and $3 \times$ FLAG-SUMO-1 demonstrated that the slowermigrating band is absent in cells expressing $\mathrm{Pax} 8^{\mathrm{K} 309 \mathrm{R}}$ and $3 \times$ FLAG-SUMO-1 (Fig. 2B). At difference, transfection experiments with $\operatorname{Pax} 8^{\mathrm{K} 91 \mathrm{R}}$, a mutant in lysine 91, demonstrated that the lysine 91 is not necessary for SUMO attachment. In fact, as shown in Fig. 2C, cells expressing $\mathrm{Pax} 8^{\mathrm{K} 91 \mathrm{R}}$ and $3 \times$ FLAG-SUMO-1 present the slower migrating band at the same extent as wild-type Pax8.

To further confirm this data, we have generated a double mutant named $\mathrm{Pax}_{8}{ }^{\mathrm{K} 2}$ in which lysines 91 and 309 were both replaced with arginine. Figure 2D shows that the sumoylation pattern of Pax8 was completely lost when the cells were transfected with this mutant. These results confirm that the major acceptor site for Pax8 sumoylation is the lysine 309 . These observations are in agreement with those of the SUMO plot prediction, that indicated the lysine 309 as a high score site for sumoylation.

To demonstrate that the higher-molecular-weight band indeed corresponds to sumoylated Pax8, we have transfected HeLa cells with the expression vectors encoding for Pax 8 or PAX $8{ }^{\mathrm{K} 309 \mathrm{R}}$ and $3 \times$ FLAG-SUMO1. Cell lysates were immunoprecipitated with an antiFLAG-agarose affinity gel and then analyzed by western blot with a polyclonal antibody against Pax8. As shown in Fig. 3, the higher-molecular-weight band was co-immunoprecipitated by anti-FLAG agarose affinity gel only in the extract prepared from cells expressing Pax8, further demonstrating that the sumoylation site in Pax8 is the lysine 309.

\section{PIASy is a SUMO ligase for Pax8}

There are three families of SUMO E3 ligases. One of these consists of the members of the PIAS family that were originally identified as transcriptional coregulators of the JAK-STAT pathway. They contain a predicted RING-finger-like structure and bind to Ubc9 and selected SUMO target proteins. Mammals have at least five members: PIAS1, PIAS3, PIASxa, PIASxb, and PIASy (Schmidt \& Muller 2003, Shuai \& Liu 2005).

To investigate whether coexpression of one of the SUMO E3 ligases could increase Pax8 sumoylation, we cotransfected HeLa cells with the expression vectors encoding Pax8, $3 \times$ FLAG-SUMO- 1 or $3 \times$ FLAG-SUMO$\Delta$ gly, and T7-PIASy. $3 \times$ FLAG-SUMO- $\Delta$ gly is a mutated form of SUMO lacking the C-terminal glycine required for the attachment to the substrates. As shown in Fig. 4, a slower-migrating band is readily detected upon transfection of $3 \times$ FLAG-SUMO-1 (lane 2), and the intensity of the band is significantly increased by the
A

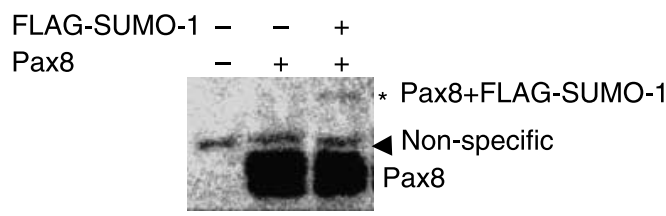

C

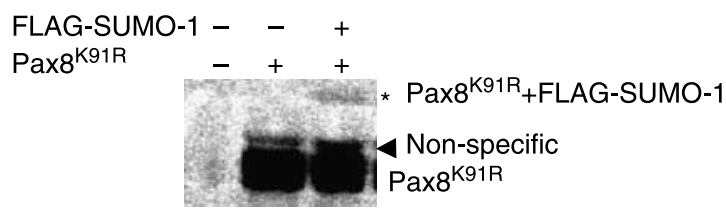

B

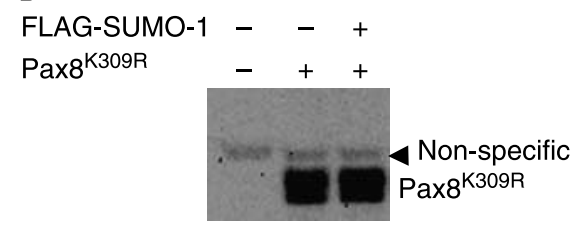

D

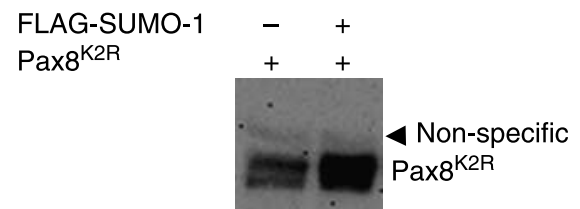

Figure 2 Modification of Pax8 with SUMO. (A) Pax8, (B) Pax8 ${ }^{\mathrm{K} 309 R}$, (C) Pax8 $8^{\mathrm{K} 91 \mathrm{R}}$ and (D) Pax8 $8^{\mathrm{K} 2 \mathrm{R}}$ were transfected in HeLa cells with or without a vector expressing $3 \times$ FLAG-SUMO-1. Forty-eight hours after transfection, protein extracts were lysed directly in the loading buffer containing the SUMO-isopeptidase inhibitor NEM $10 \mathrm{mM}$ and analyzed by western-blot with a polyclonal antibody against Pax8. The asterisk indicates an additional slower-migrating band that is compatible with a single SUMO molecule attachment. 


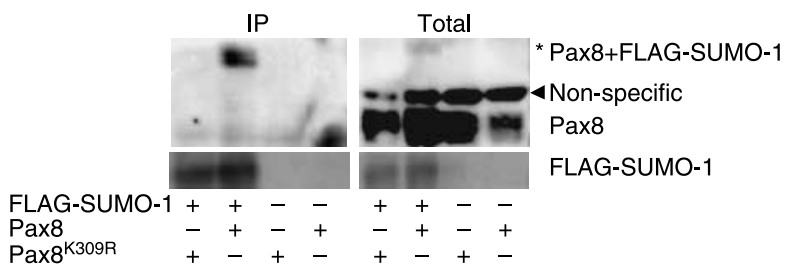

Figure 3 Immunoprecipitation of Pax8 with SUMO-1. Pax8 or Pax8 ${ }^{\mathrm{K} 309 \mathrm{R}}$ were transfected in HeLa cells with or without a vector expressing $3 \times$ FLAG-SUMO-1. Forty-eight hours after transfection, cell lysates were immunoprecipitated with an anti-FLAGagarose affinity gel and then analyzed by western blot with a polyclonal antibody against Pax8. The asterisk indicates the higher-molecular-weight band co-immunoprecipitated by antiFLAG agarose affinity gel only in the extract prepared from cells expressing Pax8 (left). Pax8 and 3×FLAG-SUMO were also detected in total lysates (right).

coexpression of PIASy (lane 3), indicating that PIASy may function as an E3 ligase for Pax8. The slowermigrating band was not visible when $\operatorname{Pax} 8$ was cotransfected with the $3 \times$ FLAG-SUMO- $\Delta$ gly mutant that is unable to bind the specific substrates (lane 4 ).

In addition, upon cotransfection of PIASy a fraction of Pax 8 conjugated to endogenous SUMO could also be detected (lane 5). By contrast, there is no evidence for sumoylation of $\operatorname{Pax} 8^{\mathrm{K} 309 \mathrm{R}}$, even in the presence of high levels of PIASy (data not shown).

These data exclude the existence of latent low-affinity sumoylation sites and further confirm that lysine 309 is the only site required for SUMO attachment to Pax8.

We next asked whether PIASy might physically interact with Pax8. To address this point, we

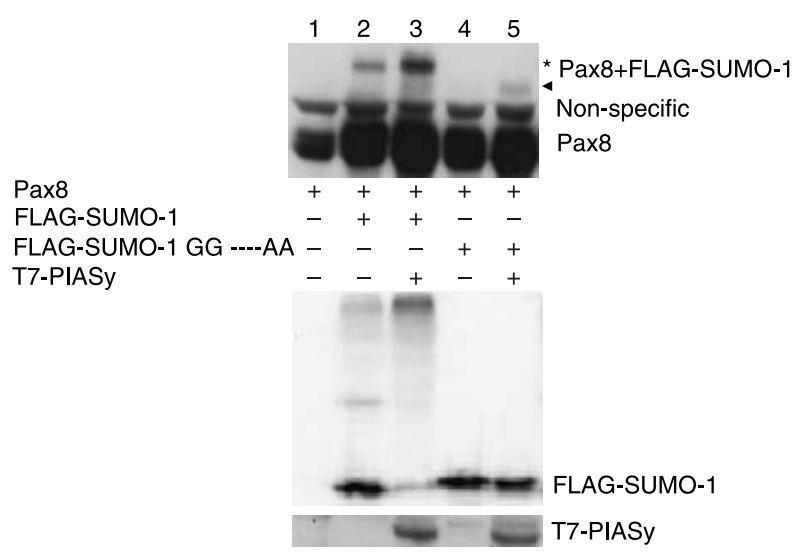

Figure 4 PIASy enhances Pax8 sumoylation. Expression vectors encoding Pax8, T7-PIASy, $3 \times$ FLAG-SUMO- 1 or $3 \times$ FLAG-SUMO$\Delta$ gly were transfected in HeLa cells as indicated. Forty-eight hours after transfection, protein extracts were lysed directly in the loading buffer containing the SUMO-isopeptidase inhibitor NEM $10 \mathrm{mM}$ and analyzed by western-blot with a polyclonal antibody against Pax8 (upper panel). The asterisk indicates the sumoylated form of Pax8. The arrow indicates a fraction of Pax 8 conjugated to endogenous SUMO detected in cells expressing PIASy (lane 5). Protein extracts were also analyzed by western-blot with monoclonal antibodies against FLAG and T7 respectively (lower panel). cotransfected HeLa cells with the expression vectors encoding $3 \times$ FLAG-Pax 8 and T7-PIASy. Cell lysates were immunoprecipitated with an anti-T7 antibody and then analyzed by western blot with a monoclonal antibody against the FLAG epitope. Figure 5 shows that PIASy immunoprecipitates with Pax8, revealing an interaction between these two proteins. Moreover, this interaction is not dependent in the presence of sumoylatable lysine, since also $\operatorname{Pax} 8^{\mathrm{K} 309 \mathrm{R}}$ is able to bind PIASy (data not shown).

\section{Pax8 accumulates in SNBs}

We next investigated the functional role of Pax8 sumoylation. Previous studies correlated SUMO modification with the recruitment of target proteins to specialized subnuclear domains. To address this issue, we transiently expressed $3 \times$ FLAG-SUMO- 1 and Pax 8 in HeLa cells and we followed their intracellular localization by confocal fluorescence microscopy. Double immunolabeling experiments with anti-FLAG and antiPax8 antibodies revealed that majority of the cells show a nuclear localization of PAX8 and SUMO-1 with a diffuse distribution, excluding areas that resemble nucleoli. Furthermore, immunofluorescence analysis revealed the presence of nuclear dots of different sizes that are positive for SUMO1 staining. Some of these dots, usually those of larger size, are also positive for Pax8 staining (Fig. 6A).

We also performed double labeling experiments for the detection of Pax8 in combination with the PML using anti-Pax 8 and anti-PML antibodies. As shown in Fig. 6B, Pax8 and PML do not colocalize, but some PML bodies are adjacent to Pax 8 nuclear dots. Recently, in differentiated neuron-like UR61 cells, it has been

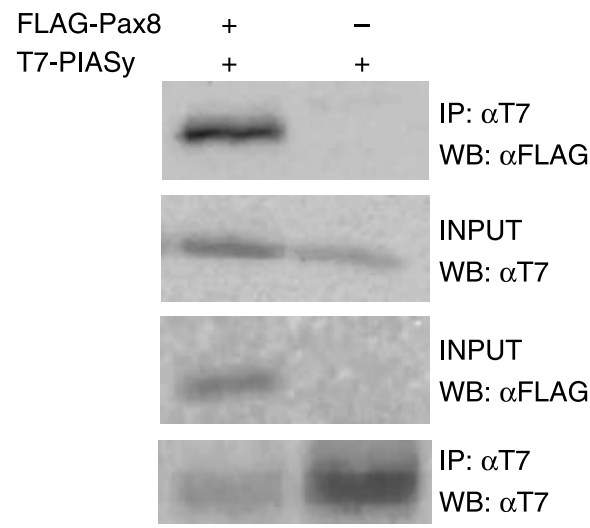

Figure 5 Interaction between PIASy and Pax8. HeLa cells were transfected with expression vectors encoding $3 \times$ FLAG-Pax 8 and T7-PIASy. Forty-eight hours after transfection, cells were lysated in RIPA buffer and were immunoprecipitated with a monoclonal antibody to T7 and immunoblotted with an antibody to the FLAG epitope. Expression of $3 \times$ FLAG-Pax8 and T7-PIASy was also analyzed in total lysates. 


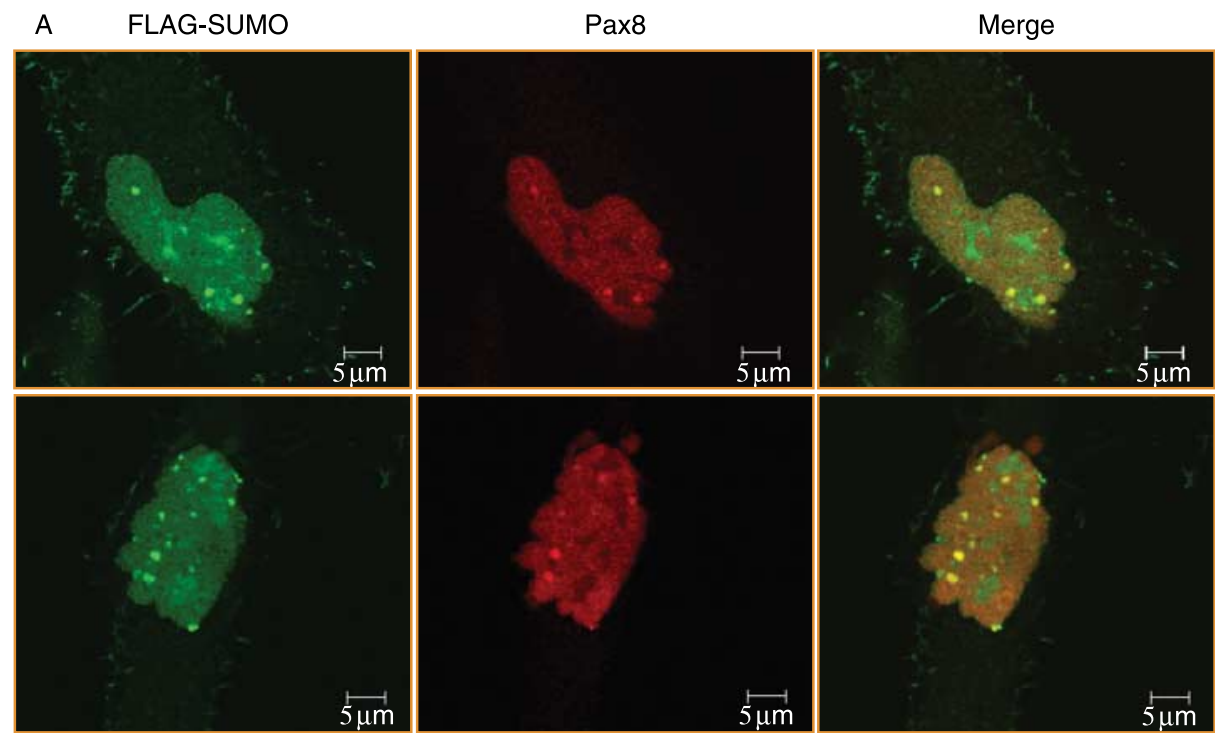

B
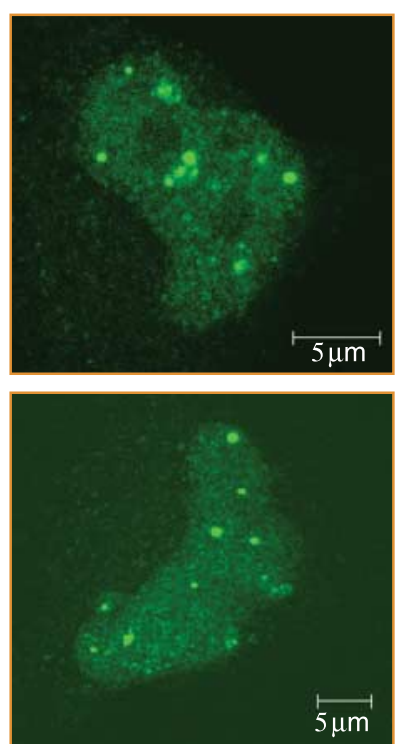

C FLAG-SUMO- $\Delta$ gly

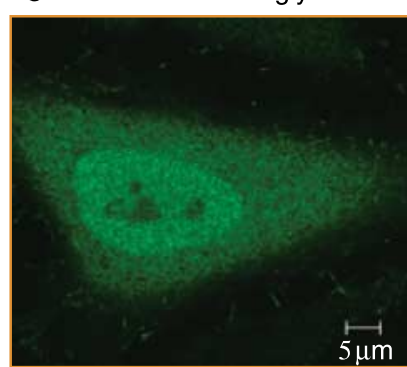

Pax8
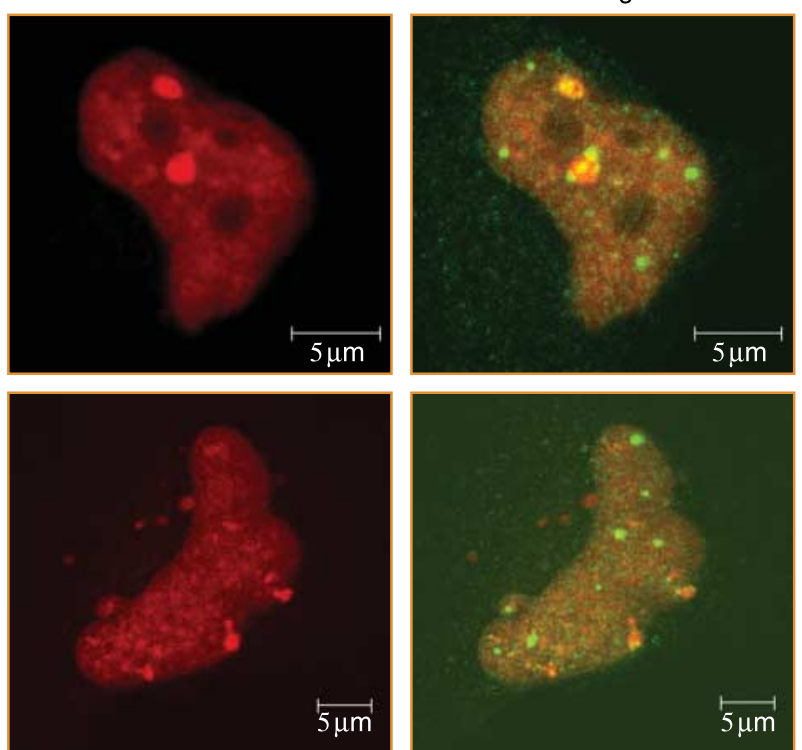

Pax8

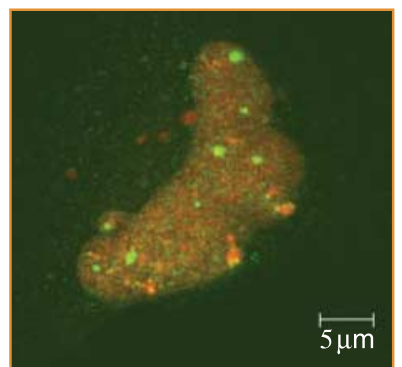

Merge
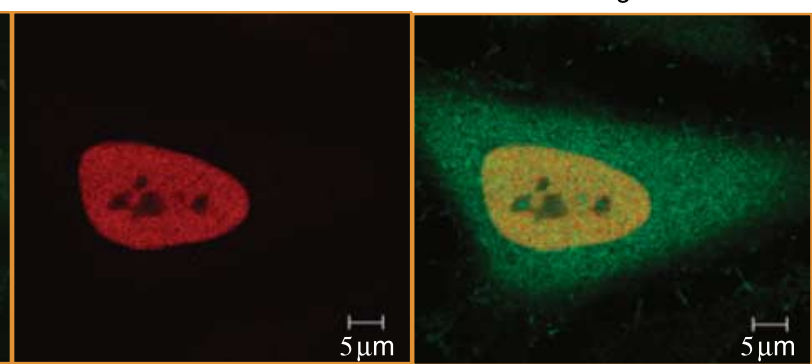

Figure 6 Pax8 accumulates in SUMO nuclear bodies. HeLa cells were transfected with Pax 8 and (A) $3 \times$ FLAG-SUMO-1 or (C) $3 \times$ FLAG-SUMO- $\Delta$ gly. At $48 \mathrm{~h}$ post-transfection, cells were processed for double immunofluorescence analysis with (A) monoclonal anti-FLAG antibody and polyclonal anti-Pax8 antibody, (B) monoclonal anti-PML antibody and polyclonal anti-Pax8 antibody and (C) anti-FLAG and antiPax8 antibodies. Samples were analyzed by confocal fluorescence microscopy. 
demonstrated that the presence of a new category of nuclear bodies called SUMO-1 nuclear bodies (SNBs), which differ from PML bodies by their large size and absence of PML protein (Navascues et al. 2007). It has been proposed that SNBs are sites of sumoylation playing a role in the control of the nucleoplasmic concentration of nuclear proteins by recruiting them in a transcription-free compartment (Navascues et al. 2007). Our results suggest that Pax 8 and SUMO-1 accumulate in PML-negative nuclear bodies that are likely to be SNBs.

To assess if SUMO-1 needs to be in an active form in order to localize in SNBs we transiently expressed $3 \times$ FLAG-SUMO- $\Delta$ gly and Pax 8 in HeLa cells and we followed their intracellular localization by fluorescence microscopy (Fig. 6C). The mutated form of $3 \times$ FLAG-SUMO- $\Delta$ gly showed a diffuse nucleoplasmic localization without a subnuclear concentration in nuclear bodies. These data indicate that only the active conjugating form of SUMO-1 is concentrated in SNBs.

To investigate the presence of SNBs in thyroid cells, we transfected PC Cl3 differentiated rat thyroid cells with $3 \times$ FLAG-SUMO- 1 and we performed double immunolabeling experiments with anti-FLAG and anti-Pax 8 antibodies to reveal the localization of endogenous Pax8. The confocal fluorescence microscopy analysis revealed, as already observed in HeLa cells, the presence of nuclear bodies positive for Pax8 and SUMO-1 indicating that the transcription factor Pax8 is targeted to SNBs also in thyroid cells
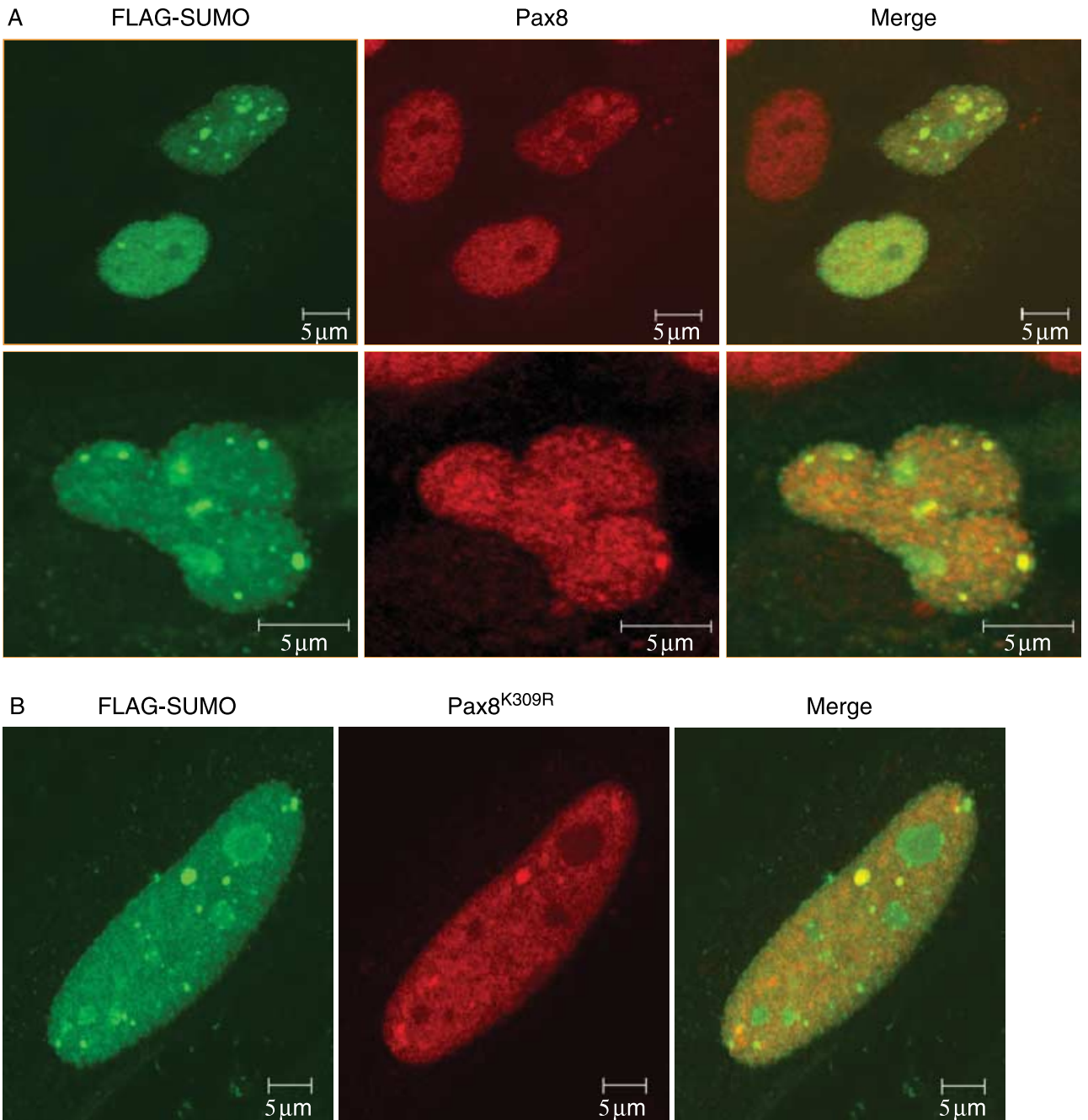

$\operatorname{Pax} 8^{\mathrm{K} 309 \mathrm{R}}$

Merge
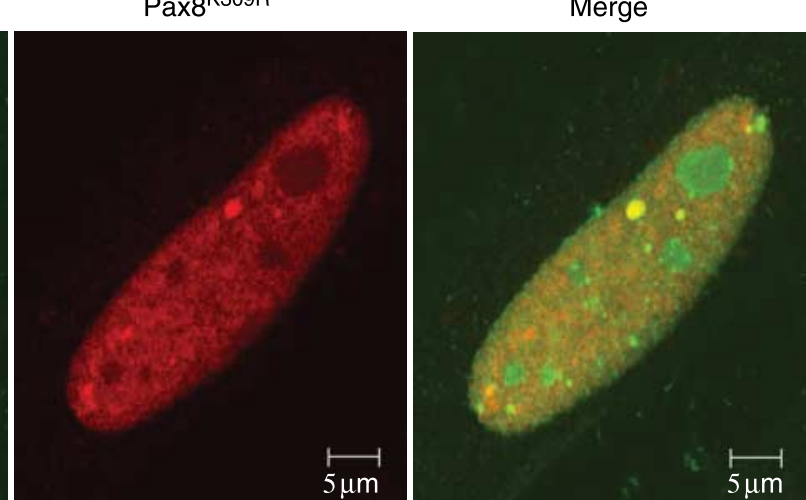

Figure 7 (A) SUMO nuclear bodies in thyroid cells. PC CL3 cells were transfected with $3 \times$ FLAG-SUMO-1 and cells were stained by immunofluorescence with monoclonal anti-FLAG antibody and polyclonal anti-Pax8 antibody. (B) Pax8 ${ }^{\mathrm{K} 309 \mathrm{R}}$ in SUMO nuclear bodies. HeLa cells were transfected with $\mathrm{Pax} 8^{\mathrm{K} 309 \mathrm{R}}$ and $3 \times$ FLAG-SUMO-1 and double immunofluorescence was performed with anti-FLAG and anti-Pax8 antibodies. Samples were analyzed by confocal fluorescence microscopy. 
(Fig. 7A). To investigate whether sumoylation of Pax8 is required for its localization in SNBs, we performed transient co-transfection in HeLa cells with the Pax8 mutant $\operatorname{Pax} 8^{\mathrm{K} 309 \mathrm{R}}$ and $3 \times$ FLAG-SUMO expression vectors. As shown in Fig. $7 \mathrm{~B}$, $\mathrm{Pax} 8^{\mathrm{K} 309 \mathrm{R}}$ colocalize with SUMO-1 in SNBs to a similar extent as wild-type Pax8. This observation suggests that PAX8 sumoylation is not necessary to target the protein to the SNBs.

\section{Sumoylation regulates Pax8 protein stability}

To analyze the effect of sumoylation on Pax8 protein stability, we transfected a wide range of concentrations of plasmid-DNA expressing Pax8 or Pax $8^{\mathrm{K} 309 \mathrm{R}}$ in HeLa cells. As shown in Fig. 8, at equal amounts of transfected DNA, Pax $8^{\mathrm{K} 309 \mathrm{R}}$ showed significantly lower protein levels in total cell extracts compared with wild-type Pax8. These results suggest that the steady-state protein levels of the mutant protein were decreased.

Successively, we asked whether reduced protein stability might explain the decreased steady-state levels of the PAX8 ${ }^{\mathrm{K} 309 \mathrm{R}}$ protein.

To address this question, HeLa cells transfected with wild-type $\operatorname{Pax} 8$ or with $\operatorname{Pax} 8^{\mathrm{K} 309 \mathrm{R}}$ were treated with cycloheximide (CHX) for different times to block protein synthesis. In this way, it has been possible to follow the steady-state levels of proteins that had been synthesized before CHX treatment. After treatment, cells were lysed and proteins were subjected to western blot with a polyclonal antibody against Pax8. The quantification of the Pax 8 band by densitometry
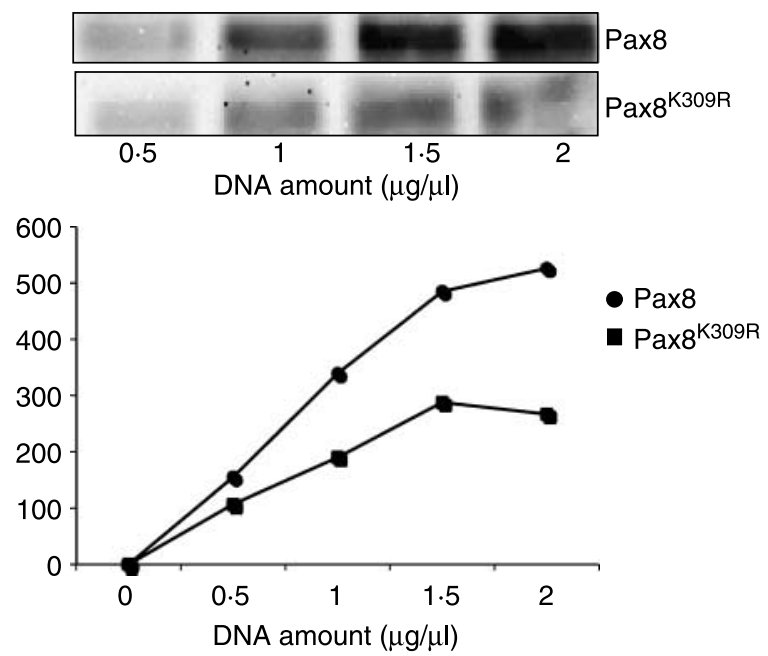

Figure $8 \mathrm{Pax} 8^{\mathrm{K} 309 \mathrm{R}}$ protein stability compared with wild-type Pax8. HeLa cells were transfected with Pax8 or Pax $8^{\mathrm{K} 309 \mathrm{R}}$ as indicated. Forty-eight hours after transfection, cells were lysed and proteins were analyzed by western blotting using anti-Pax8 antibody (upper panel). Blots were analyzed by densitometry and results of three independent experiments were plotted (bottom panel). revealed that the levels of Pax 8 were more abundant than those of the Pax8 mutant (Fig. 9A). In addition, HeLa cells were cotransfected with PAX8 or Pax $8^{\mathrm{K} 309 \mathrm{R}}$ and with or without $3 \times$ FLAG-SUMO and $12 \mathrm{~h}$ after transfection the cells were treated with $\mathrm{CHX}$ for different times. The proteins were subjected to western blot with a polyclonal antibody against Pax8 and the quantification of the Pax 8 band by densitometry revealed that the levels of wild-type Pax 8 were increased when expressed with SUMO, while the same effect did not occur for Pax $8^{\mathrm{K} 309 \mathrm{R}}$ (Fig. 9B).

All together, these experiments suggest that Pax8 stability is controlled by sumoylation and that the Pax 8 mutant, which cannot be sumoylated, is degraded more rapidly.

\section{Transcriptional activity of $\operatorname{Pax}^{\mathrm{K} 309 \mathrm{R}}$}

To elucidate whether the attachment of SUMO to Pax8 might affect Pax8 transcriptional activity, we performed transactivation assays in HeLa cells co-transfecting the reporter construct CP5-CAT, an artificial promoter responsive only to Pax8, together with the expression vectors encoding for Pax8 or Pax $8^{\mathrm{K} 309 \mathrm{R}}$. As expected, the cotransfection of wild-type Pax8 led to a significant activation of the CP5-CAT promoter (Fig. 10A). Inversely, the cotransfection of $\mathrm{Pax} 8^{\mathrm{K} 309 \mathrm{R}}$ showed a decreased transcriptional activation of the same promoter. Successively, we performed a western blot with a polyclonal antibody against Pax8 on the protein extracts of the transactivation assay described above. As shown in Fig. 10A, the results demonstrated that the decreased transcriptional activity of $\mathrm{Pax} 8^{\mathrm{K} 309 \mathrm{R}}$ corresponds to a reduced protein expression. To evaluate the ability of PAX $8^{\mathrm{K} 309 \mathrm{R}}$ to bind to DNA, we carried out electrophoretic mobility-shift assay (EMSA) using protein extracts prepared from HeLa cells transfected with the vectors encoding for $\mathrm{Pax} 8$ or $\mathrm{Pax} 8^{\mathrm{K} 309 \mathrm{R}}$. Figure 10B shows that $\operatorname{Pax} 8$ and $\operatorname{Pax} 8^{\mathrm{K} 309 \mathrm{R}}$ bind with a similar affinity to the double-stranded oligonucleotide containing Pax 8 consensus sequence. Taken together, these data further confirm that sumoylation affects Pax8 protein stability while it doesn't modify its transcriptional activity or its ability to bind DNA.

\section{Discussion}

Despite the critical role played by Pax 8 in thyroid organogenesis and in the regulation of the thyroid differentiated phenotype, very little is known about its post-translational modifications and of the mechanisms by which these modifications may regulate its transcriptional activity. In this work, we focused our attention on the SUMO modification that in the past few years has emerged as an important regulator 
A Time of $\mathrm{CHX}$ treatment $(\mathrm{h})$
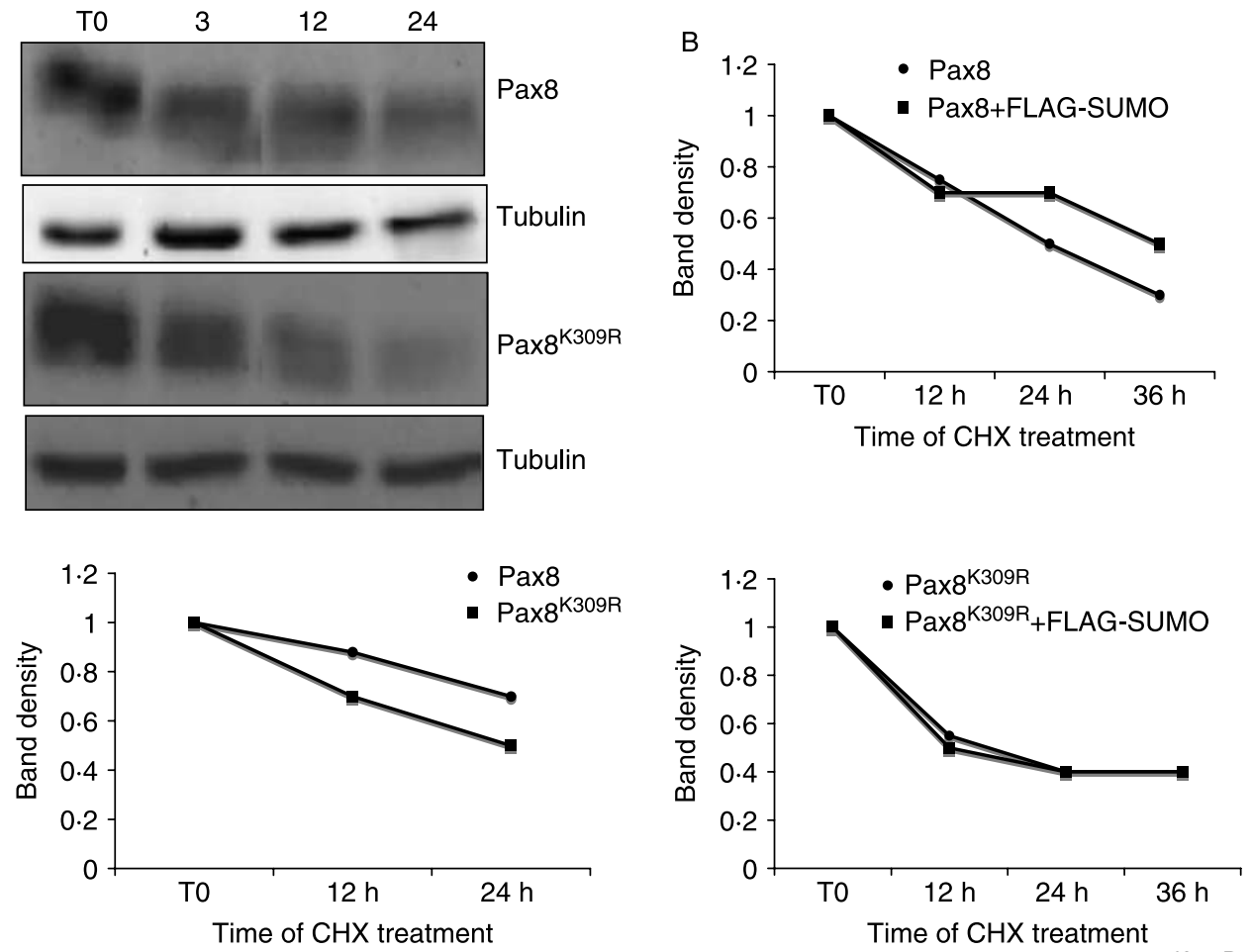

Figure 9 Increased stability of Pax8 compared with the sumoylation-deficient mutant Pax $8^{\mathrm{K} 309 \mathrm{R}}$. (A) HeLa cells were transfected with Pax 8 or Pax $8^{\mathrm{K} 309 \mathrm{R}}$ and $12 \mathrm{~h}$ after transfection, cells were treated with $100 \mu \mathrm{g} / \mathrm{ml}$ cycloheximide (CHX) for the indicated time periods. Cells were lysed and proteins were analyzed by western blotting using anti-Pax 8 antibody. The hybridization with $\alpha$-tubulin assessed the protein uniform loading and integrity (upper panel). Western blots were analyzed by densitometry and results of three independent experiments ( \pm S.E.M.) were plotted (bottom panel). (B) HeLa cells were transfected with Pax8 or Pax $8^{\mathrm{K} 309 \mathrm{R}}$ and $3 \times \mathrm{FLAG-SUMO1.} \mathrm{Twelve} \mathrm{hours} \mathrm{after} \mathrm{transfection,} \mathrm{cells} \mathrm{were}$ treated with $100 \mathrm{mg} / \mathrm{ml}$ cycloheximide $(\mathrm{CHX})$ for the indicated time periods. The results of western blots were analyzed by densitometry and results of three independent experiments ( \pm S.E.M.) were plotted.

of diverse pathways and activities including transcription factor stability, protein-protein interaction and subcellular localization of target proteins (Meulmeester \& Melchior 2008).

We demonstrated that Pax8 is a substrate for SUMO modification, and we showed that lysine 309 , which lies within a perfectly matching consensus sequence $\psi \mathrm{KxE}$, is the residue involved in this post-translational modification. Mutagenesis of lysine 309 completely abolishes Pax8 modification by SUMO, thus indicating that lysine 309 is the only sumoylation site in Pax8.

To further demonstrate that Pax8 is a substrate for sumoylation, we investigated whether coexpression of a SUMO E3 ligase was able to increase the fraction of sumoylated Pax8 and we identified PIASy as a potent SUMO ligase for Pax8. At the same time, we also showed that PIASy binds to Pax8 and that this interaction is not dependent on the presence of the sumoylatable lysine.

There is no simple way to predict which functional consequences the sumoylation of a given target might have. In fact, sumoylation is a highly dynamic process and its outcomes are multiple, ranging from changes in cellular localization to modification in the activity or stability of the modified protein. We investigated all these aspects in order to assign a functional role to Pax8 sumoylation. We addressed the question of the subcellular localization and we demonstrated that mutation of Pax8 sumoylation sites does not affect its subcellular localization. Interestingly, immunofluorescence analysis, in both non-thyroid and thyroid cells, revealed the presence of large size nuclear dots positive for Pax8 and SUMO staining. In parallel, we also demonstrated that Pax8 forms nuclear bodies colocalized with SUMO at the same extent of wild-type Pax8. Hence, we propose that Pax8 is targeted and sumoylated into the SNBs. It has been proposed that these structures might regulate the nucleoplasmic concentration of transcription factors by SUMO trapping (Navascues et al. 2007). Therefore, the macromolecular assembly of SUMO complexes into SNBs might lead to the stabilization of transcriptional factors in a nuclear compartment refractory to degradation. In this context, 


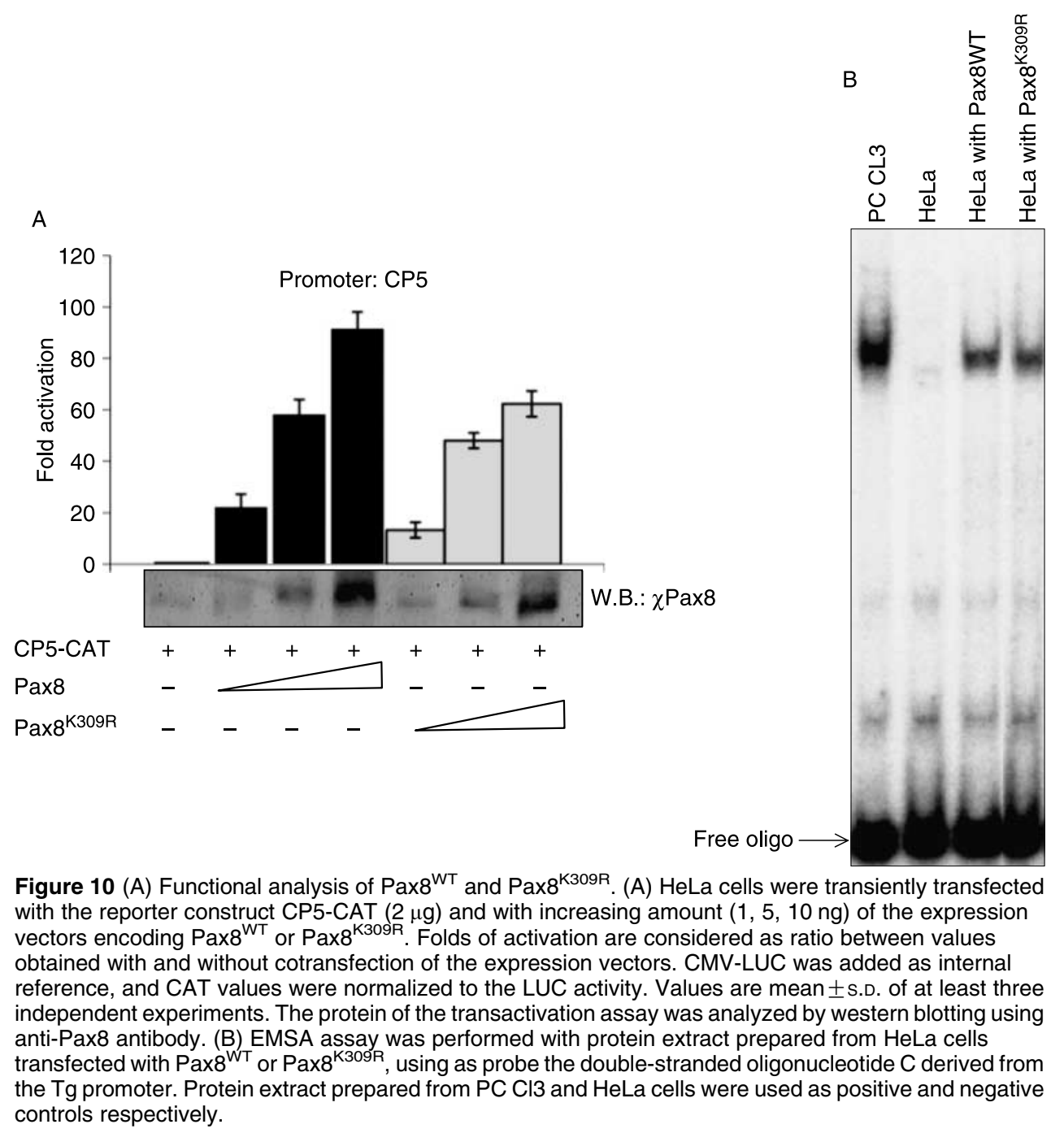

we hypothesized that $\mathrm{Pax} 8^{\mathrm{K} 309 \mathrm{R}}$ is targeted into SNBs as well as Pax8, but since it cannot be sumoylated it is degraded more rapidly. To assess this hypothesis, we investigated whether sumoylation could affect Pax8 protein stability and our data clearly show that indeed it does. The data of the role of sumoylation in the control of Pax8 stability were further confirmed by transactivation assays in HeLa cells. In fact, we demonstrated that the decreased transcriptional activity of $\mathrm{Pax} 8^{\mathrm{K} 309 \mathrm{R}}$ corresponds to a reduced protein expression, confirming that sumoylation affects protein stability of Pax 8 and doesn't modify the transcriptional activity or the ability to bind DNA of Pax8.

In conclusion, we propose that Pax8 is sumoylated in SNBs that are nuclear bodies distinct from PML bodies, in which nuclear proteins may be organized and assembled. The macromolecular assembly of SUMO complexes into SNBs might lead to the stabilization of transcriptional regulators in a nuclear compartment refractory to degradation. The transcription factor Pax 8 has been shown to be critically involved in determination and maintenance of the differentiation state of thyrocytes. Consequently, we think that the nucleoplasmic concentration of Pax8 may be a relevant aspect in the regulation of the expression of the thyroidspecific genes that are considered as markers of the differentiated phenotype. Interestingly, a variable regulation of Pax8 sumoylation might explain the marked differences in the phenotype of the subjects with Pax8 loss-of-function mutations. In fact, the penetrance/expressivity of Pax 8 mutations has been shown to be variable, suggesting that other factors like a dominant negative effect or haploinsufficiency may modulate the phenotypic expression (Damante 1998, Macchia et al. 1998, de Sanctis et al. 2004). Hence, we would like to propose that a different state of Pax8 sumoylation caused by genetic and environmental factors could be an explanation for the phenotypic 
variability that occurs in patients carrying the mutations. Nevertheless, additional studies are now required to identify the physiological stimulus that controls Pax8 sumoylation. It is known that the expression of Pax8 is under the control of TSH/cAMP and that this control operates at a transcriptional level. Thus, it is possible that the same TSH/cAMP pathway or other physiological signals may be involved in the regulation of Pax 8 posttranslational modifications, modulating for example its nucleoplasmic concentration.

\section{Declaration of interest}

The authors declare that there is no conflict of interest that could be perceived as prejudicing the impartiality of the research reported.

\section{Funding}

This work was supported by a grant from the Associazione Italiana per la Ricerca sul Cancro (AIRC) to M Z. T Di Palma has been supported by a fellowship from the Federazione Italiana per la Ricerca sul Cancro (FIRC).

\section{Acknowledgements}

The authors gratefully acknowledge $\mathrm{Dr} \mathrm{G}$ del Sal for providing the T7PIASy expression vector and for helpful discussion and the Service of Molecular Biology (SBM) of the Stazione Zoologica A. Dohrn of Naples for techinal assistance.

\section{References}

Ambesi-Impiombato FS \& Coon HG 1979 Thyroid cells in culture. International Review of Cytology 10 163-172.

Berlingieri MT, Portella G, Grieco M, Santoro M \& Fusco A 1988 Cooperation between the polyomavirus middle-T-antigen gene and the human c-myc oncogene in a rat thyroid epithelial differentiated cell line: model of in vitro progression. Molecular and Cellular Biology 8 2261-2266.

Cardone L, Hirayama J, Giordano F, Tamaru T, Palvimo IJ \& SassoneCorsi P 2005 Circadian clock control by SUMOylation of BMAL1. Science 309 1390-1394.

Collavin L, Gostissa M, Avolio F, Secco P, Ronchi A, Santoro C \& Del Sal G 2004 Modification of the erythroid transcription factor GATA-1 by SUMO-1. PNAS $1018870-8875$.

Dahle O, Andersen TO, Nordgard O, Matre V, Del Sal G \& Gabrielsen OS 2003 Transactivation properties of c-Myb are critically dependent on two SUMO-1 acceptor sites that are conjugated in a PIASy enhanced manner. European Journal of Biochemistry 270 1338-1348.

Damante G 1998 Thyroid defects due to Pax8 gene mutations. European Journal of Endocrinology 139 563-566.

Girdwood D, Bumpass D, Vaughan OA, Thain A, Anderson LA, Snowden AW, Garcia-Wilson E, Perkins ND \& Hay RT 2003 P300 transcriptional repression is mediated by SUMO modification. Molecular Cell 11 1043-1054.

Hay RT 2005 SUMO: a history of modification. Molecular Cell 18 1-12.

Hay RT 2007 SUMO-specific proteases: a twist in the tail. Trends in Cell Biology 17 370-376.

Heun P 2007 SUMOrganization of the nucleus. Current Opinion in Cell Biology 19 350-355.
Holmstrom S, Van Antwerp ME \& Iniguez-Lluhi JA 2003 Direct and distinguishable inhibitory roles for SUMO isoforms in the control of transcriptional synergy. PNAS 100 15758-15763.

Hunter T 2007 The age of crosstalk: phosphorylation, ubiquitination, and beyond. Molecular Cell 28 730-738.

Kambe F \& Seo H 1996 Mediation of the hormone- and serumdependent regulation of thyroglobulin gene expression by thyroidtranscription factors in rat thyroid FRTL-5 cells. Journal of Endocrinology 150 287-298.

Kuo HY, Chang CC, Jeng JC, Hu HM, Lin DY, Maul GG, Kwok RP \& Shih HM 2005 SUMO modification negatively modulates the transcriptional activity of CREB-binding protein via the recruitment of Daxx. PNAS 102 16973-16978.

Macchia PE, Lapi P, Krude H, Pirro MT, Missero C, Chiovato L, Souabni A, Baserga M, Tassi V, Pinchera A et al. 1998 PAX8 mutations associated with congenital hypothyroidism caused by thyroid dysgenesis. Nature Genetics 19 83-86.

Mansouri A, Chowdhury K \& Gruss P 1998 Follicular cells of the thyroid gland require Pax8 gene function. Nature Genetics 19 87-90.

Mascia A, Nitsch L, Di Lauro R \& Zannini M 2002 Hormonal control of the transcription factor Pax8 and its role in the regulation of thyroglobulin gene expression in thyroid cells. Journal of Endocrinology 172 163-176.

Melchior F 2000 SUMO-nonclassical ubiquitin. Annual Review of Cell and Developmental Biology 16 591-626.

Meulmeester E \& Melchior F 2008 Cell biology: SUMO. Nature $\mathbf{4 5 2}$ 709-711.

Missero C, Cobellis G, De Felice M \& Di Lauro R 1998 Molecular events involved in differentiation of thyroid follicular cells. Molecular and Cellular Endocrinology 140 37-43.

Muller S, Berger M, Lehembre F, Seeler JS, Haupt Y \& Dejean A 2000 c-Jun and p53 activity is modulated by SUMO-1 modification. Journal of Biological Chemistry 275 13321-13329.

Muller S, Hoege C, Pyrowolakis G \& Jentsch S 2001 SUMO, ubiquitin's mysterious cousin. Nature Reviews. Molecular Cell Biology 2 202-210.

Navascues J, Bengoechea R, Tapia O, Vaque JP, Lafarga M \& Berciano MT 2007 Characterization of a new SUMO-1 nuclear body (SNB) enriched in pCREB, CBP, c-Jun in neuron-like UR61 cells. Chromosoma 116 441-451.

Di Palma T, Nitsch R, Mascia A, Nitsch L, Di Lauro R \& Zannini M 2003 The paired domain-containing factor Pax8 and the homeodomaincontaining factor TTF-1 directly interact and synergistically activate transcription. Journal of Biological Chemistry 278 3395-3402.

Pasca di Magliano M, Di Lauro R \& Zannini M 2000 Pax8 has a key role in thyroid cell differentiation. PNAS 97 13144-13149.

Plachov D, Chowdhury K, Walther C, Simon D, Guenet JL \& Gruss P 1990 Pax8, a murine paired box gene expressed in the developing excretory system and thyroid gland. Development 110 643-651.

Poleev A, Fickenscher H, Mundlos S, Winterpacht A, Zabel B, Fidler A, Gruss P \& Plachov D 1992 PAX8, a human paired box gene: isolation and expression in developing thyroid, kidney and Wilms' tumors. Development 116 611-623.

Ross S, Best JL, Zon LI \& Gill G 2002 SUMO-1 modification represses Sp3 transcriptional activation and modulates its subnuclear localization. Molecular Cell 10 831-842.

de Sanctis L, Corrias A, Romagnolo D, Di Palma T, Biava A, Borgarello G, Gianino P, Silvestro L, Zannini M \& Dianzani I 2004 Familial PAX8 small deletion (c.989_992delACCC) associated with extreme phenotype variability. Journal of Clinical Endocrinology and Metabolism 89 5669-5674.

Schmidt D \& Muller S 2003 PIAS/SUMO: new partners in transcriptional regulation. Cellular and Molecular Life Sciences 60 2561-2574.

Seeler JS \& Dejean A 2003 Nuclear and unclear functions of SUMO. Nature Reviews. Molecular Cell Biology 4 690-699.

Shuai K \& Liu B 2005 Regulation of gene-activation pathways by PIAS proteins in the immune system. Nature Reviews. Immunology $\mathbf{5}$ 593-605. 
Song J, Durrin LK, Wilkinson TA, Krontiris TG \& Chen Y 2004 Identification of a SUMO-binding motif that recognizes SUMOmodified proteins. PNAS 101 14373-14378.

Stuart ET \& Gruss P 1996 PAX: developmental control genes in cell growth and differentiation. Cell Growth and Differentiation 7 405-412.

Tirard M, Almeida OF, Hutzler P, Melchior F \& Michaelidis TM 2007 Sumoylation and proteasomal activity determine the transactivation properties of the mineralocorticoid receptor. Molecular and Cellular Endocrinology 268 20-29.

de Wet JR, Wood KV, DeLuca M, Helinski DR \& Subramani S 1987 Firefly luciferase gene: structure and expression in mammalian cells. Molecular and Cellular Biology 7 725-737.
Zannini M, Francis-Lang H, Plachov D \& Di Lauro R 1992 Pax-8, a paired domain-containing protein, binds to a sequence overlapping the recognition site of a homeodomain and activates transcription from two thyroid-specific promoters. Molecular and Cellular Biology 12 4230-4241.

Zhao J 2007 Sumoylation regulates diverse biological processes. Cellular and Molecular Life Sciences 64 3017-3033.

Received in final form 29 September 2008

Accepted 30 October 2008

Made available online as an Accepted Preprint 30 October 2008 